\title{
EVALUATION OF THE THERAPEUTIC EFFECTS OF SABBAGH UNIVERSAL SPRING COMPARED TO CLASS II ELASTICS IN THE TREATMENT OF CLASS II DIVISION 1 PATIENTS
}

\author{
Dina Osman ElAbbasy*
}

\begin{abstract}
Aim: The purpose of this clinical prospective study was to compare between class II elastics and a "no compliance" fixed functional appliance therapy SUS ${ }^{2}$ (Sabbagh Universal Spring 2, Dentaurum, Ispringen, Germany) with respect to the skeletal and dental changes following correction of Class II division 1 malocclusion in adolescent subjects compared to a matched untreated Class II control group.
\end{abstract}

Materials and Methods: The study was carried out on 30 female patients with Class II, division 1 malocclusion with a mean age of 14.5 years old. All patients were matched regarding cause of malocclusion with mandibular retrognathia being the main source. The growth pattern was normal or low-angle. The subjects were randomly allocated into 3 groups, each group comprising 10 patients: Group 1 (SUS ${ }^{2}$ ), Group 2 (Class II elastics) and Group C (Control group). Treatment/ observation were carried out for an average period of 8 months. The appliances were removed when a Class I dental relationship was achieved. Pre- and post-treatment/observation lateral cephalograms were analyzed to determine the treatment effects. Student's paired t-test and Chi-squared test were used for intragroup and intergroup comparisons.

Results: There was an overjet reduction in both groups with a marked improvement towards normal Class I occlusion. Skeletal effects were more marked in SUS ${ }^{2}$ group compared to Class II elastics group with a mean decrease in angles SNA , ANB and MP/SN $\left(-0.89 \pm 0.9^{\circ},-1.97 \pm 1.2^{\circ}\right.$, and $-0.8 \pm 1.3^{\circ}$ respectively) while an increase was evident for angle SNB and Co-Gn $\left(1.08 \pm 0.5^{\circ}\right.$ and $2.1 \pm 0.9 \mathrm{~mm}$ ) respectively. All linear skeletal parameters showed statistically significant changes in the $\mathrm{SUS}^{2}$ group except MP/SN while in the Class II elastics no statistically significant changes were observed except for angle MP/SN that showed statistically significant increase $\left(1.5 \pm 1.2^{\circ}\right)$. U1/SN angle showed statistically significant decrease in both treatment groups $(\mathrm{P}<0.01)$ while L1/ MP showed statistically significant increases but were more pronounced in Class II elastics group $(\mathrm{P}<0.01)$.

Conclusions: SUS2 is more effective than Class II elastics in the treatment of skeletal Class II maloccluion as it yields mild but more favorable skeletal results and less dentoalveolar side effects.

* Lecturer, Department of Orthodontics, Cairo University 


\section{INTRODUCTION}

Skeletal class II malocclusion is the most prevalent sagittal skeletal discrepancy and is considered a major indication for orthodontic treatment. It represents about $21 \%$ of the Egyptian adult population and about $15-20 \%$ in Caucasian population (Hanandeh and El-Bialy, 2010). The characteristics of this malocclusion include skeletal as well as dental factors that can vary in severity. Skeletal factors include mandibular retrognathism and/or maxillary prognathism. Mandibular retrognathism is more common and constitutes one third of the population (McNamara, 1981). Mandibular retrognathism may be due to a small mandible, posterior placement of condyle in the glenoid fossa or a functional retrusion. Treatment of Class II malocclusion depends on the severity of the problem and the age of the patient. Various orthodontic appliances and techniques have been used for treatment including intra-arch and interarch appliances, different extraction patterns, extra-oral appliances and orthognathic surgery (Bishara et al, 1997 and Janson et al, 2006).

Removable functional appliances are indicated for use in younger ages (prepubertal) and promote supplemental mandibular growth and remodeling of the condyle. However, they require significant patient cooperation whilst fixed functional appliances (FFA) could be used in older ages (such as adolescence) and provide constant forces without the need for patient cooperation (Abd el-magid , 1995; Konik et al, 1997 and Covell et al, 1999). Various contradictory reports have been found in the literature regarding the true effects of fixed functional appliances on the mandible.

Fixed functional appliances are compliancefree interarch appliances. Although they display proven efficiency for correcting Class II Division
1 malocclusions, conclusions differ with regards to the relative degree of skeletal versus dentoalveolar effects. In addition to their dentoalveolar effects in Class II correction, some authors reported that they also exert variable skeletal effects (Weiland \& Bantleon, 1995 and Stucki \& Ingervall, 1998). They also eliminate the patient compliance factor. There is considerable debate as to whether FFAs can stimulate mandibular growth and potentially lead to last-ing skeletal changes.

Only a few types of fixed functional appliances have been investigated. The SUS ${ }^{2}$ (Sabbagh Universal Spring) is one of the newest FFA and has been claimed to give predictable results and use of residual growth even beyond the pubertal growth spurt. It is also believed to shorten treatment time, easy to install and less prone to breakages (Sabbagh, 2006 and Hanandeh \& El-Bialy, 2010).

Class II elastics or intermaxillary elastics are well documented means of correcting Class II malocclusion and are believed to produce mainly dentoalveolar changes. The use of Class II elastics or light wire Begg technique in the treatment of Class II malocclusion has always been an issue of controversy. Although class II elastics are effective for treatment ,their side effects have long been demonstrated by several authors (Nelson et al, 1999, Combrink et al, 2006 and Uzel et al, 2007) ranging from lower incisor proclination, upper incisor extrusion, lower molar extrusion, clockwise rotation of the mandibular plane as well as need of utmost patient compliance for maximum benefit. Poor patient cooperation can lead to poor treatment outcome and prolonged treatment time (Beckwith et al, 1999 and Skidmore et al, 2006).

Hence this study was conducted to evaluate and compare the skeletal and dental effects of fixed functional appliance (Sabbagh Universal Spring) 
and Class II elastics in the treatment of skeletal Class II malocclusion.

\section{MATERIALS AND METHODS}

The study sample consisted of 30 female patients selected from the outpatient clinic of the department of Orthodontics, Cairo University. They fulfilled the following criteria (Figure 1):

1- Age range 13-15.5 years (postpubertal or post -peak) CVM stage (CS 5-6) identified from digital lateral cephalograms (Baccetti et al, 2005).

2 - Class II Division 1 malocclusion due to mandibular retrognathia (SNB angle $<=77$ )

3- Full permanent dentition

4- Class II end-on molar relationship

5- Half-unit Class II canine relationship

6- Minimal crowding in the dental arches

8- Increased overjet at least $6 \mathrm{~mm}$

9- Maxillary incisor normal or slight proclination - normally inclined or upright mandibular incisors

10- Horizontal to average growth pattern

11- No history of previous orthodontic treatment or extraction

12- No craniofacial syndromes or systemic diseases

11- No TMJ symptoms

The subjects were randomly allocated into three groups, each group consisting of 10 patients $(n=10)$ : Group 1 ( $\left.\mathrm{SUS}^{2}\right)$, Group 2 (Class II elastics) and Group 3 (Control).

The aim of the research and the procedures were explained to the patients and parents and a written consent was signed to participate in the study. The research was approved by the institutional ethics committee.

For every patient before the beginning of treatment, full orthodontic records were obtained including photographs, radiographs as well as plaster study models. Lateral cephalograms were taken in centric occlusion before the start of orthodontic treatment (T0), 2 days before placement of the SUS ${ }^{2}$ and Class II elastics (T1) and at the end of treatment after removal of the fixed functional appliance and elastics (T2) using the Instrumentarium Imaging Orthoceph OC100 type cephalostate with an anode-to-midsubject distance of $152.5 \mathrm{~cm}$ and a midsubject-to-film distance of $14.6 \mathrm{~cm}$ with a standard magnification of $8 \%$. For control subjects, the lateral cephalograms were taken at the beginning and end of the observation periods (T1 and T2) at the same timing of the treatment groups. They were then imported to Dolphin imaging software version 11.0 for lateral cephalometric analysis, whereby the anatomical landmarks and reference lines were digitally identified and tracing was done through the software. The measuring points and reference lines were those defined by Steiner (Proffit, 1999 and McNamara, 1984) (Figures 1,3).

The landmarks identified were as follows:

SNA: Position of the maxillary jaw base relative to the anterior cranial base

SNB: Position of the mandibular jaw base relative to the anterior cranial base

ANB: Position of the maxillary and mandibular skeletal bases relative to eachother

MP/SN: Mandibular plane inclination relative to the $\mathrm{SN}$ plane

Pt A-NaP: Linear distance from point A to Nasion perpendicular, relates maxilla to cranial base.

Pog-NaP: Linear distance from Pogonion to Nasion perpendicular, relates mandible to cranial base.

Co-Gn: Mandibular length from condylion to gnathion. 


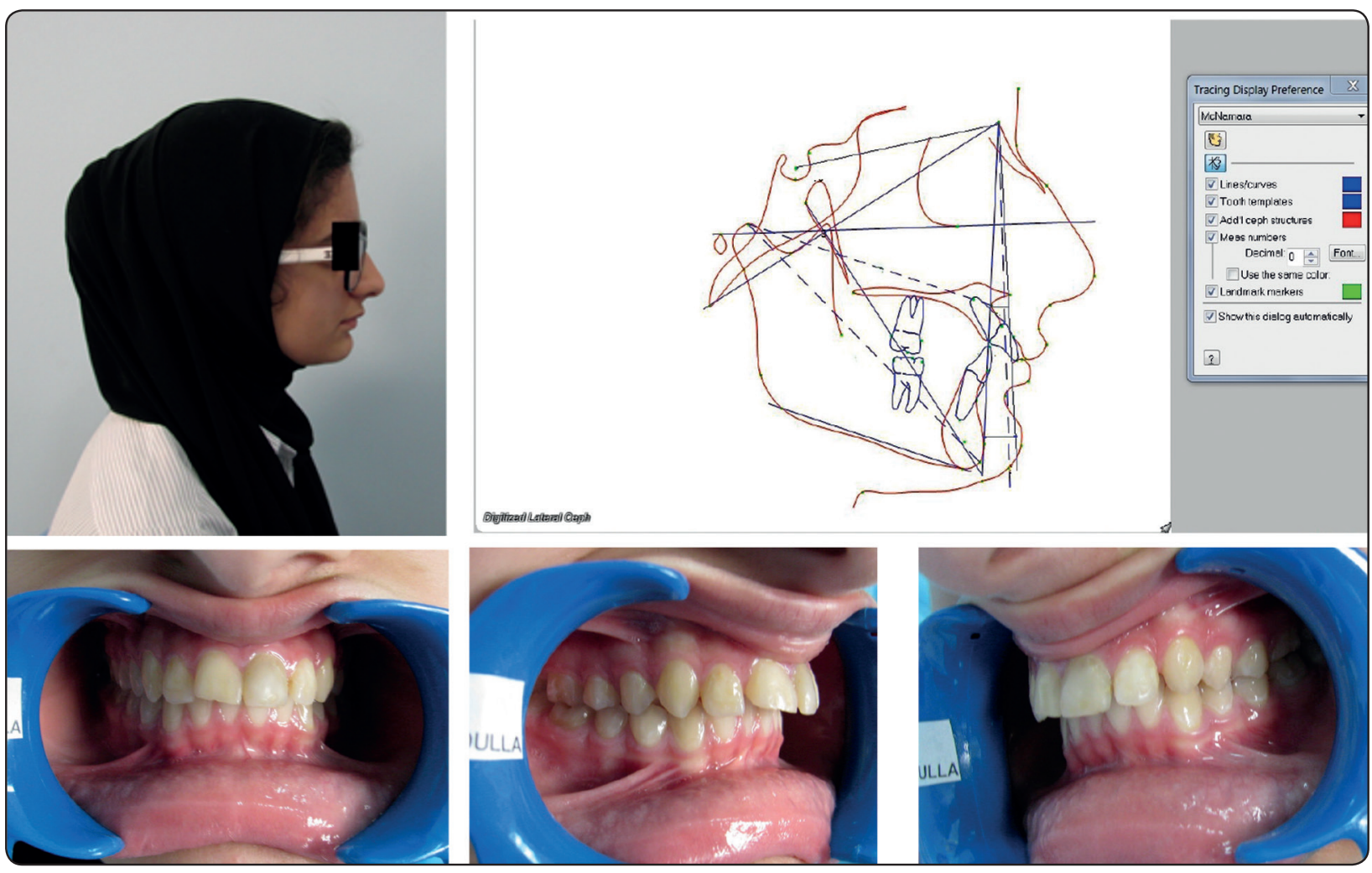

Fig. (1): Pretreatment photos (T0) and cephalometric tracing of patient treated by SUS ${ }^{2}$ (T1)

U1/SN: Inclination of the long axis of the upper incisor with the SN plane.

L1/MP: inclination of the long axis of the lower incisors with the mandibular plane (Go-Me).

The groups were matched with regards to Class II malocclusion severity by comparing the initial mean values of angles ANB and MP-SN.

For Groups 1 and 2, the treatment mechanics were the same with regards to bracket prescription including (MBT prescription 0.022-in slot preadjusted appliance) (Ortho Organizers, San Marcos, Calif) and stage of archwires. The patients were fitted with upper and lower orthodontic fixed appliances and progressed with alignment and leveling (0.014 Ni-Ti, 0.016 stainless steel, $0.019 \times 0.025$ stainless steel cinched back to minimize anchorage loss) to fit the $\mathrm{SUS}^{2}$.

The SUS2 is a telescopic device that combines the advantages of both Herbst and Jasper Jumper and overcomes their disadvantages. It consists of a telescopic rod located in a guide tube. Inside the tube there is a spring that can be activated according to the level of forces desired. There is an Omega loop that is attached in the accesory tube of the upper first permanent molar band and in the lower arch the device is attached between the canine and first premolar with a SUS ${ }^{2}$ arch adapter screw (Figure 2).

After measuring the correct size of the appliance, the SUS ${ }^{2}$ was fixed according to the manufacturer's instructions. In the upper arch a transpalatal arch was fitted to control maxillary first molars transverse expansion. The SUS $^{2}$ appliances were activated every eight weeks by placing a spacer (closed) spring, with steps not more than $4 \mathrm{~mm}$ for larger overjets. Gradual expansion of the maxillary arch was performed to coordinate the upper and lower arches while sagittal correction was achieved 


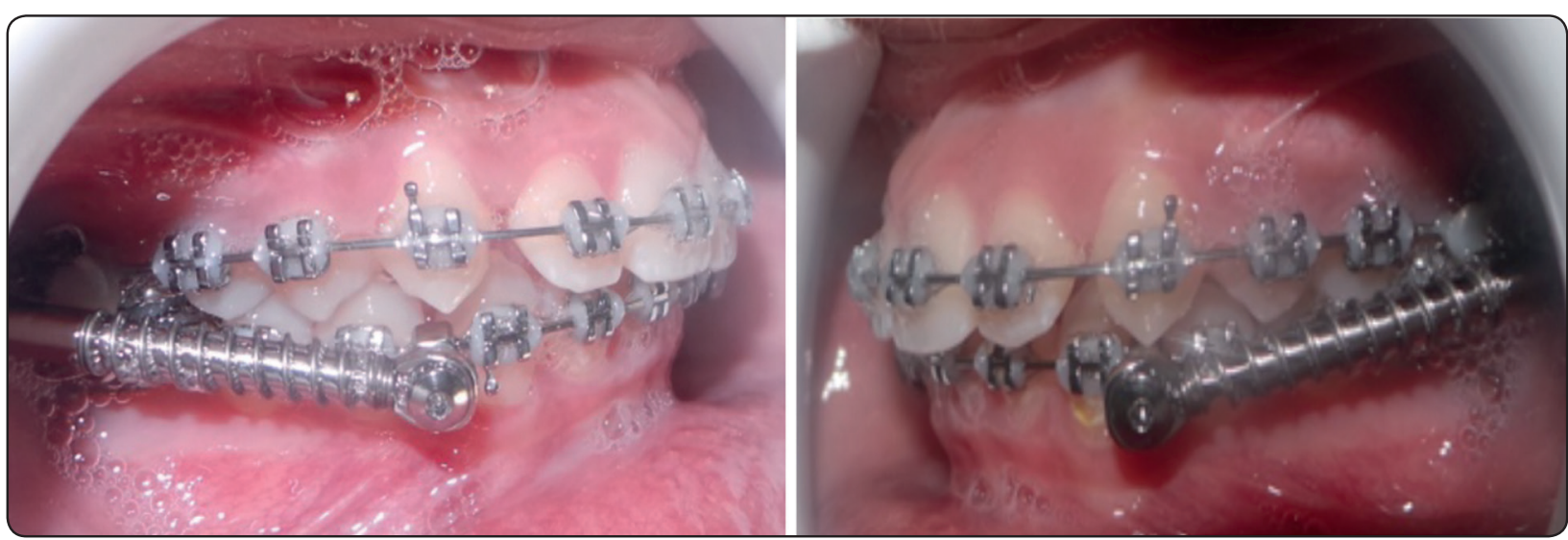

Fig. (2) Sabbagh Universal Spring (SUS2) inserted intraorally

by mandibular advancement. Patients were asked to report to the clinic immediately in case any breakages or detachments occur. Class II elastics were placed from the maxillary permanent canines bracket hooks to the mandibular first permanent molars on upper and lower $0.019 \times 0.025$ stainless steel archwires. The elastics diameter was $3 / 16$ inches and force of $3.5 \mathrm{oz}$. Patients were instructed to wear the elastics $24 / 7$ even during eating (Figure 4).

The average active treatment duration was 8 months for both treatment groups as well as observation period for control group. SUS ${ }^{2}$ and Class II elastics were removed when a Class I or overcorrected Class I canine and molar relationship /end-to-end incisor relationship was achieved.

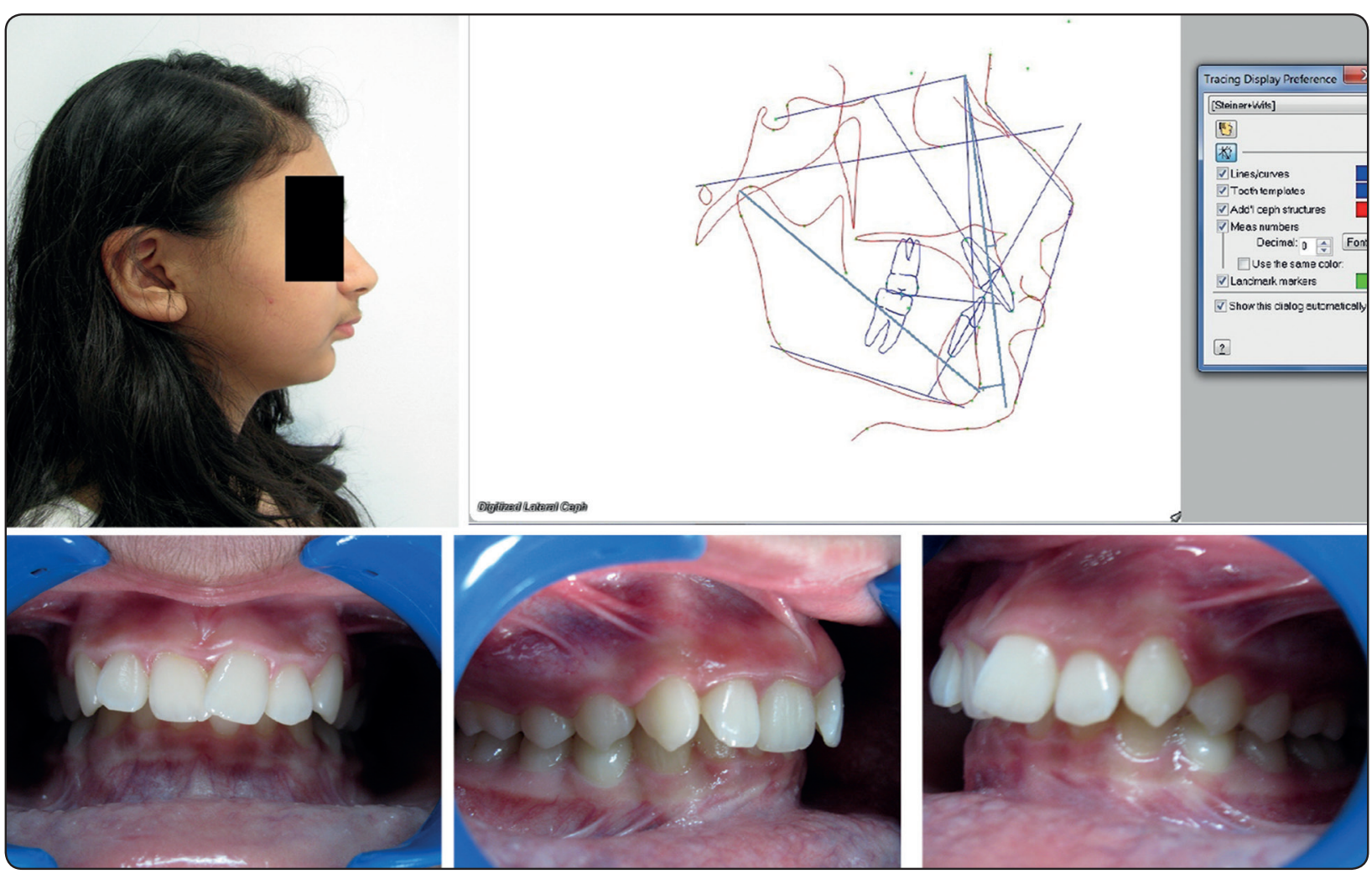

Fig. (3): Pretreatment photos (T1) and lateral cephalometric tracing of patient treated with Class II elastics (T1) 


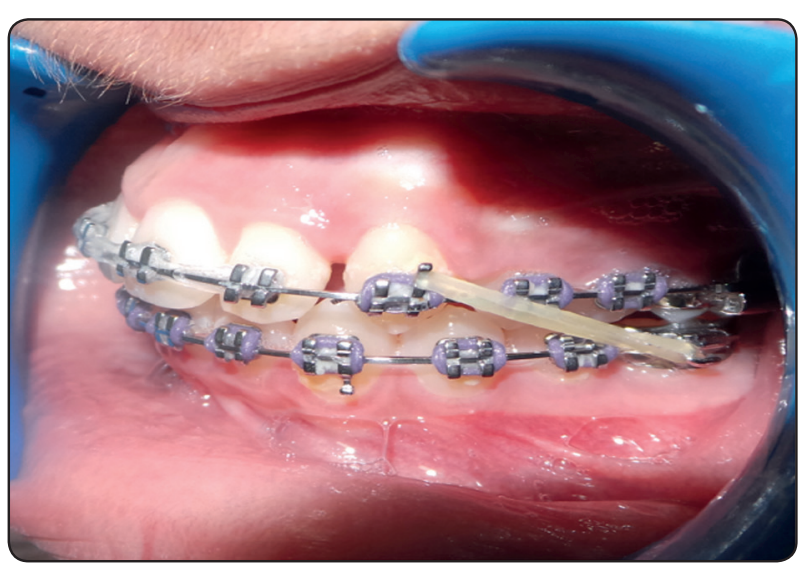

Fig. (4): Midstage treatment with Class II elastics

\section{Statistical methods}

All Data were collected, tabulated and subjected to statistical analysis. Statistical analysis was performed by SPSS in general (version 17), while Microsoft office Excel was used for data handling and graphical presentation.

Quantitative variables were described by the Mean, Standard Deviation (SD), the Range (Minimum - Maximum),Standard Error (SE) and 95\% confidence interval of the mean.

Qualitative categorical variables were described by proportions and Percentages.

Shapiro-Wilk test of normality was used to test normality hypothesis of all quantitative variables for further choice of appropriate parametric and non parametric tests. Mostly the variables were found normally distributed allowing the use of parametric tests. Paired samples $t$ test was used for comparing the Post and Pre within each group. Independent samples $t$ test was used for comparing the difference (Post-Pre) between the two groups . Chi-squared test was applied for 2 by 2 contingency table.

Significance level was considered at $\mathrm{P}<0.05$ (S); while for $\mathrm{P}<0.01$ was considered highly significant (HS). Two Tailed tests were assumed through out the analysis for all statistical tests.

\section{RESULTS}

A total of 30 participants were enrolled in this study and they were randomly and equally distributed between the three groups with 10 patients in each group.

All subjects were corrected to dental Class I relation in a mean treatment duration of 8 months 5 days \pm ( 2 months 3 days) for SUS $^{2}$ group (Figure 5 ) and 8 months 2 days \pm (1 month 5 days) for the Class II elastics group (Figure 6). Normal overjet and overbite were achieved.

No significant differences existed between the mean ages and treatment/observation durations which showed the homogeneity between the 3 groups (Table 1).

The mean changes in the values of the cephalometric variables at the $\mathrm{T} 1$ insertion of SUS $^{2}$ and Class II elastics and those at the end of the treatment/observation period $\mathrm{T} 2$ are shown in (Table 2) .There were statistically significant differences between $\mathrm{T} 1$ and $\mathrm{T} 2$ for all cephalometric variables in $\mathrm{SUS}^{2}$ group except angle MP/SN which showed statistically insignificant decrease. No statistically significant changes were recorded for all the skeletal parameters in Class II elastics group except for mandibular plane inclination which revealed statistically significant increase. However, there were statistically significant changes for the dentoalveolar measurements $(\mathrm{P}<0.001)$. The changes in the control group were insignificant and were mainly due to minor growth changes which were matched to reference values from the study of Weiland et al, 1997.

When SUS 2 and Class II elastics were compared there were statistically significant differences between all the variables where angles SNA and ANB showed a statistically significant decrease $\left(-0.89 \pm 0.9^{\circ}\right.$ and $\left.-1.97 \pm 1.2^{\circ}\right)(\mathrm{P}<0.05$ and $\mathrm{P}<0.01)$ respectively in SUS ${ }^{2}$ compared to Class II elastics $\left(-0.3 \pm 1.12^{\circ}\right.$ and $\left.-0.5 \pm 0.73^{\circ}\right)$. Therefore, the apical 


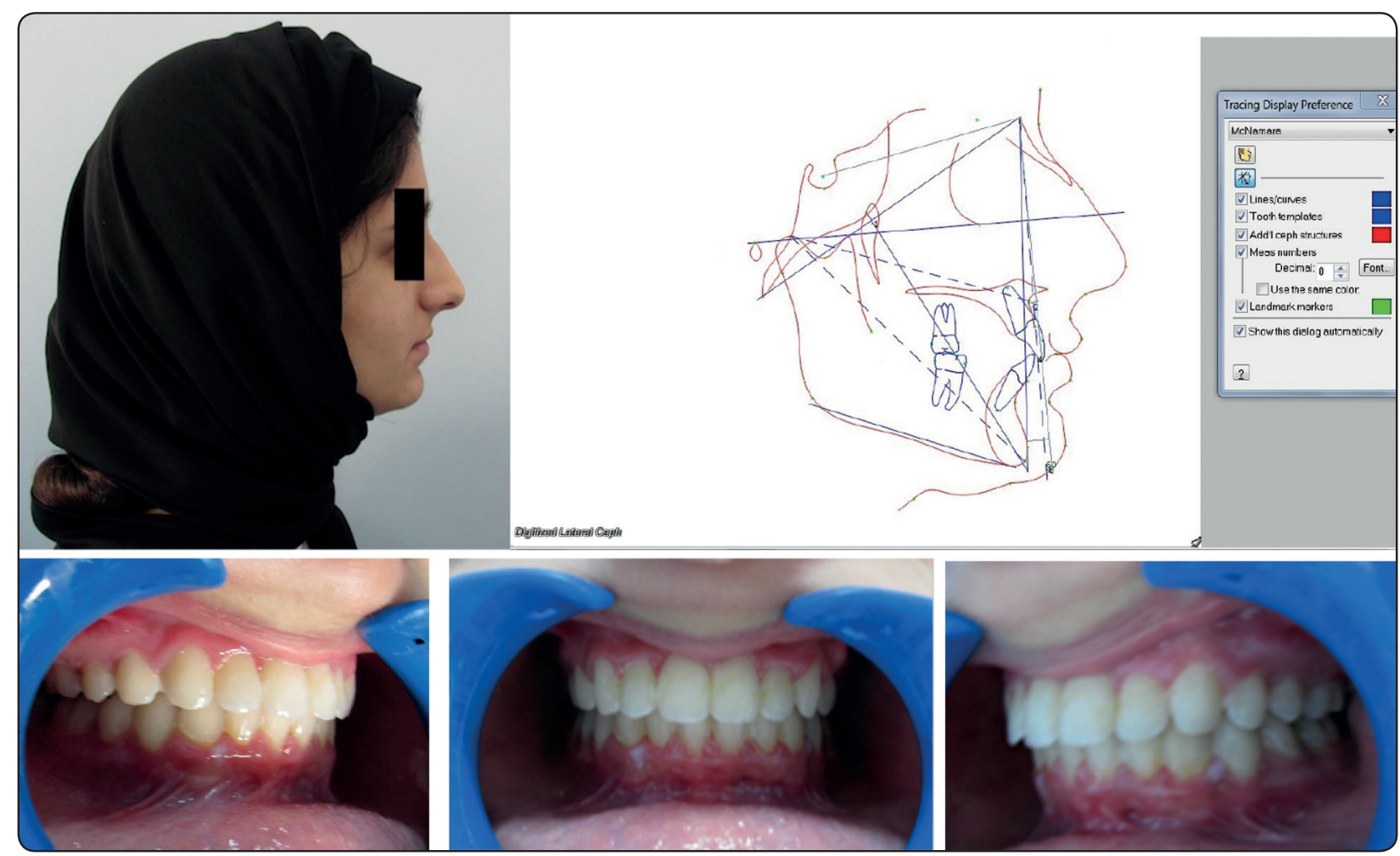

Fig. (5): Post-treatment photos and lateral cephalogram in the SUS2 treatment group

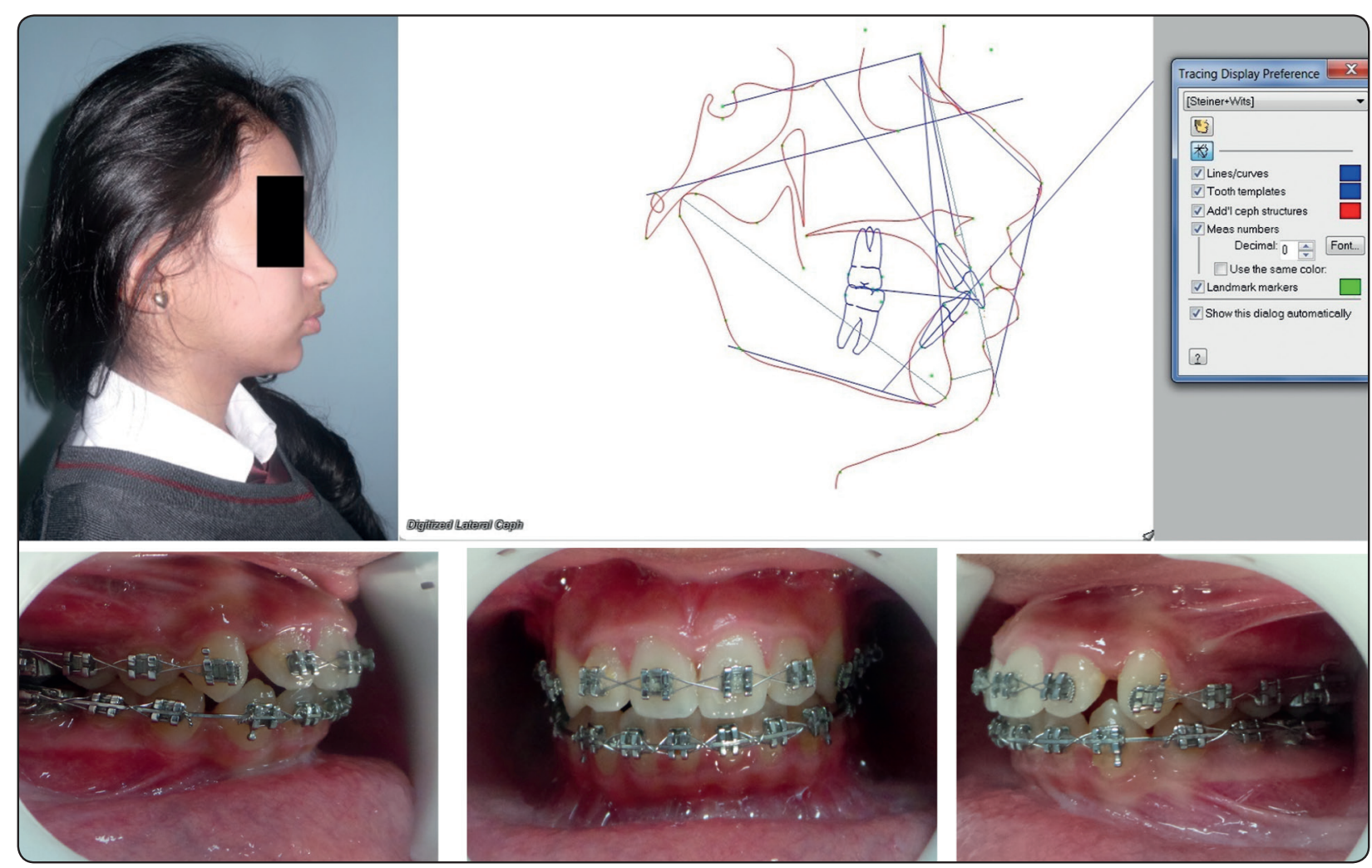

Fig. (6): Post-treatment photos and lateral cephalometric tracing in the Class II elastics treatment group 
base changes which depict the maxillomandibular relationship revealed greater improvement in the SUS $^{2}$ group than in the Class II elastics group. Angle SNB showed statistically significant increase in $\operatorname{SUS}^{2}\left(1.08 \pm 0.5^{\circ}\right)(\mathrm{P}<0.01)$ compared to Class II elastics $\left(0.2 \pm 0.82^{\circ}\right)$. Angle $\mathrm{MP} / \mathrm{SN}$ or the vertical jaw relationship showed statistically significant decrease $\left(-0.8 \pm 1.3^{\circ}\right) \quad(\mathrm{P}<0.01)$ in $\mathrm{SUS}^{2}$ group compared to a statistically significant increase in Class II elastics group $\left(1.5 \pm 1.2^{\circ}\right)$

Regarding linear measurements, there were statistically significant decrease in $\mathrm{Pt} A-\mathrm{NaP}$ $(-0.5 \pm 1.6 \mathrm{~mm})(\mathrm{P}<0.01)$ in $\mathrm{SUS}^{2}$ compared to Class II elastics $(-0.1 \pm 0.6 \mathrm{~mm})$ which confirms the restraining effect of the appliance on forward maxillary growth. Statistically significant differences were also found between SUS $^{2}$ and Class II elastics in the measurements Pog-NaP and Co-Gn where they revealed significant changes in $\mathrm{SUS}^{2}$ group $(1.6 \pm 1.2 \mathrm{~mm}$ and $2.1 \pm 0.9 \mathrm{~mm})$ respectively compared to $(0.3 \pm 1.5 \mathrm{~mm}$ and $0.3 \pm 0.9 \mathrm{~mm})$ in Class II elastics group $(\mathrm{P}<0.01)$.

Regarding dentoalveolar changes, they were significantly higher in Class II elastics compared to SUS $^{2}$ where upper incisors displayed more retroclination and lower incisors showed a higher degree of proclination in Class II elastics $\left(-7.2 \pm 2.45^{\circ}\right.$ and $\left.8 \pm 3.1^{\circ}\right)$ respectively $(\mathrm{P}<0.01)$ compared to $\left(-5.72 \pm 1.56^{\circ}\right.$ and $\left.6.2 \pm 2.1^{\circ}\right)$ respectively in $\mathrm{SUS}^{2}$ group.

As shown in (Table 3) when SUS ${ }^{2}$ group was compared to Control group, there were statistically significant changes in all the angular and linear measurements except MP/SN which was statistically insignificant. Angles SNA, ANB, U1/SN and linear measurement Pt A-NaP(mm) showed significant decreases when compared with the Control group $(\mathrm{P}<0.05, \mathrm{P}<0.01)$. On the other hand angle $\mathrm{SNB}$ , L1/MP, Pog-NaP(mm) and Co-Gn(mm) showed significant increases compared with the Control group $(\mathrm{P}<0.01, \mathrm{P}<0.05)$. Comparison between the Class II elastics and control group revealed no statistically significant changes for all the angular and linear measurements $(\mathrm{P}>0.05)$ with the exception of the upper and lower incisors inclination $\left(-7.2 \pm 2.45^{\circ}\right.$ and $\left.8 \pm 3.1^{\circ}\right)$ as well as MP/SN $\left(1.5 \pm 1.2^{\circ}\right)$ compared to $\left(0.93 \pm 1.5^{\circ}, 0.25 \pm 0.9^{\circ}\right.$ and $\left.-0.43 \pm 0.6^{\circ}\right)$ respectively in Control group $(\mathrm{P}<0.05, \mathrm{P}<0.01)$.

TABLE (1) Mean age and treatment duration values between the 3 study groups

\begin{tabular}{|c|c|c|c|c|}
\hline & SUS $^{2}$ & Class II elastics & Control & P-value \\
\hline Mean age & $\begin{array}{c}14 \text { years } 5 \text { months } \pm 1 \\
\text { year }\end{array}$ & $\begin{array}{c}14 \text { years } 7 \text { months } \pm 1 \\
\text { year 3 months }\end{array}$ & $\begin{array}{c}14 \text { years } 7 \text { months } \pm 1 \\
\text { year 2 months }\end{array}$ & 0.210 \\
\hline Treatment duration & $\begin{array}{c}8 \text { months } 5 \text { days } \pm 2 \\
\text { months 3 days }\end{array}$ & $\begin{array}{c}8 \text { months 2 days } \pm 1 \\
\text { month } 5 \text { days }\end{array}$ & $\begin{array}{c}8 \text { months } 7 \text { days } \pm 2 \\
\text { months } 2 \text { days }\end{array}$ & 0.341 \\
\hline
\end{tabular}

$P>0.05$ (NS) 
TABLE (2) Mean values of cephalometric parameters at T1 and T2 for the 3 study groups

\begin{tabular}{|c|c|c|c|c|c|c|c|c|c|}
\hline \multirow{3}{*}{ Variable } & \multirow{2}{*}{\multicolumn{3}{|c|}{ SUS GroupMean \pm SD }} & \multirow{2}{*}{\multicolumn{3}{|c|}{$\begin{array}{c}\text { Class II elastics Group } \\
\text { Mean } \pm \text { SD }\end{array}$}} & \multirow{2}{*}{\multicolumn{3}{|c|}{$\begin{array}{c}\text { Control Group } \\
\text { Mean } \pm \text { SD }\end{array}$}} \\
\hline & & & & & & & & & \\
\hline & $\mathrm{T} 1$ & $\mathrm{~T} 2$ & $\begin{array}{c}\mathrm{p}- \\
\text { value }\end{array}$ & $\mathrm{T} 1$ & $\mathrm{~T} 2$ & $\begin{array}{c}\mathrm{p}- \\
\text { value }\end{array}$ & $\mathrm{T} 1$ & $\mathrm{~T} 2$ & $\begin{array}{c}\mathrm{P}- \\
\text { value }\end{array}$ \\
\hline SNA $^{\circ}$ & $81.2 \pm 1.4$ & $80.31 \pm 2.1$ & $*$ & $80.34 \pm 2.02$ & $80.04 \pm 2.5$ & -- & $81.56 \pm 2.9$ & $81.69 \pm 3.0$ & -- \\
\hline $\mathrm{SNB}^{\circ}$ & $75.63 \pm 1.1$ & $76.71 \pm 1.5$ & $*$ & $74.96 \pm 2.6$ & $75.16 \pm 2.1$ & -- & $76.08 \pm 2.5$ & $76.19 \pm 2.9$ & -- \\
\hline $\mathrm{ANB}^{\circ}$ & $5.57 \pm 0.80$ & $3.6 \pm 14$ & $*$ & $5.38 \pm 1.1$ & $4.88 \pm 1.7$ & -- & $5.60 \pm 1.4$ & $5.5 \pm 1.3$ & -- \\
\hline $\begin{array}{l}\text { Pt A to Nasion } \\
\text { perp }(\mathrm{mm})\end{array}$ & $0.6 \pm 3.2$ & $0.1 \pm 3.5$ & $*$ & $0.4 \pm 3.1$ & $0.3 \pm 2.6$ & -- & $0.7 \pm 2.8$ & $0.9 \pm 2.4$ & -- \\
\hline $\begin{array}{l}\text { Pog to Nasion } \\
\text { Perp (mm) }\end{array}$ & $-6.4 \pm 4.6$ & $-4.8 \pm 4$ & $*$ & $-5.8 \pm 3.8$ & $-5.5 \pm 4.1$ & -- & $-5.5 \pm 4.8$ & $-5.0 \pm 4.6$ & -- \\
\hline Co-Gn(mm) & $109.7 \pm 5.1$ & $111.8 \pm 5.7$ & $*$ & $107.9 \pm 4.3$ & $108.2 \pm 4.7$ & -- & $106.5 \pm 5.3$ & $107.9 \pm 4.9$ & -- \\
\hline $\mathrm{MP} / \mathrm{SN}^{\circ}$ & $29.6 \mathrm{~S} \pm 3.24$ & $28.88 \pm 3$ & -- & $31.47 \pm 2.5$ & $32.97 \pm 3.7$ & $* *$ & $31.87 \pm 3.1$ & $31.44 \pm 3.4$ & -- \\
\hline UIISN $^{\circ}$ & $104.42 \pm 2.76$ & $98.7 \pm 2.3$ & $* *$ & $103.31 \pm 4.3$ & $96.11 \pm 3.6$ & $* *$ & $103.55 \pm 4.1$ & $104.48 \pm 3.5$ & -- \\
\hline $\mathrm{LI} / \mathrm{MPo}$ & $98.86 \pm 3.5$ & $105.06 \pm 3.1$ & $* *$ & $99.81 \pm 3.8$ & $107.81 \pm 4.7$ & $* *$ & $97.81 \pm 4.2$ & $98.06 \pm 4.4$ & -- \\
\hline
\end{tabular}

$P>0.05$ NS (--), $* P<0.05, * * P<0.01$

TABLE (3) Comparison of cephalometric changes (T2-T1) among the treatment \& control groups

\begin{tabular}{|c|c|c|c|c|c|c|}
\hline & \multicolumn{3}{|c|}{ Mean $\pm \mathrm{SO}$} & \multicolumn{3}{|c|}{ P-Value } \\
\hline $\begin{array}{c}\text { Cephalometric } \\
\text { measures }\end{array}$ & SUS2 Group & $\begin{array}{c}\text { Class II elastics } \\
\text { Group }\end{array}$ & Control Group & $\begin{array}{l}\text { SUS2- } \\
\text { Control }\end{array}$ & $\begin{array}{l}\text { Class II elastics- } \\
\text { Control }\end{array}$ & $\begin{array}{c}\text { SUS2-Class II } \\
\text { elastics }\end{array}$ \\
\hline $\mathrm{SNA}^{\circ}$ & $-0.89 \pm 0.9$ & $-0.3 \pm 1.12$ & $0.13 \pm 0.9$ & $*$ & -- & $*$ \\
\hline $\mathrm{SNB}^{\circ}$ & $1.08 \pm 0.5$ & $0.2 \pm 0.82$ & $0.11 \pm 0.7$ & $* *$ & -- & $* *$ \\
\hline $\mathrm{ANB}^{\circ}$ & $-1.97 \pm 1.2$ & $-0.5 \pm 0.73$ & $-0.1 \pm 0.5$ & $* *$ & -- & $* *$ \\
\hline PtA-NaP(mm) & $-0.5 \pm 1.6$ & $-0.1 \pm 0.6$ & $0.2 \pm 0.9$ & $*$ & -- & $*$ \\
\hline Pog-NaP(mm) & $1.6 \pm 1.2$ & $0.3 \pm 1.5$ & $0.8 \pm 1.2$ & $*$ & -- & $* *$ \\
\hline Co-Gn(mm) & $2.1 \pm 0.9$ & $0.3 \pm 0.9$ & $1.1 \pm 1.4$ & $*$ & $*$ & $* *$ \\
\hline $\mathrm{MP} / \mathrm{SN}^{\mathrm{o}}$ & $-0.8 \pm 1.3$ & $1.5 \pm 1.2$ & $-0.43 \pm 0.6$ & $*$ & $* *$ & $* *$ \\
\hline $\mathrm{U} 1 / \mathrm{SN}^{\mathrm{o}}$ & $-5.72 \pm 1.86$ & $-7.2 \pm 2.45$ & $0.93 \pm 1.5$ & $* *$ & $* *$ & $* *$ \\
\hline $\mathrm{Ll} / \mathrm{MP}^{\circ}$ & $6.2 \pm 2.1$ & $8 \pm 3.1$ & $0.25 \pm 0.9$ & $* *$ & $* *$ & $* *$ \\
\hline
\end{tabular}




\section{DISCUSSION}

Numerous orthodontic techniques and appliances are available for the treatment of Class II malocclusion. Fixed functional appliances and intermaxillary or Class II elastics are typical interarch treatment modalities used for correction of Class II malocclusion.

Some authors advocated against the use of Class II elastics due to their side effects such as proclination of the lower incisors as well as extrusion of upper incisors and lower molars resulting in clockwise occlusal plane rotation (Buchner, 1949; Ellen et al, 1998 ; Nelson et al, 1999 and Jones et al, 2008). Furthermore, they mainly rely on patients' cooperation. Fixed functional appliances have been developed over the past two decades and show considerable promise in this area as they provide continuous force and do not rely on patient's compliance. This study was designed to compare between the two treatment modalities regarding the skeletal and dental effects when Class II elastics are used as the primary treatment protocol rather than an adjunctive treatment.

The current study is the first to compare therapeutic changes effected by Sabbagh Universal Spring with those induced by Class II elastics in Class II malocclusion. It has long been postulated that fixed functional appliances can substitute Class II elastics in the treatment of non-compliant patients as their effects are identical and are mainly dentoalveolar. A systematic review by Janson et al, 2013 showed that, on a long-term basis, there are no significant differences between the effects of Class II elastics and other removable or fixed functional appliances in Class II malocclusion treatment.

Therefore, this study was conducted to investigate the treatment effects of both techniques and determine whether they are comparable or not

The study sample included only female patients who were at their post-pubertal growth period. No males were included due to the different timing of growth spurts between males and females which influences the normal growth changes and may provide misleading results on the true effect of the fixed functional appliance. The inclusion of an untreated and age-matched control group in this study with similar skeletal and dental characteristics was justified in order to identify and exclude any normal growth effects throughout the treatment period.

Intervention at this stage (CVM5,CM6) is believed to benefit the patients due to the presence of residual growth even in minimal amounts. It is also believed to result in minimal relapse from growth and posttreatment dentoskeletal changes (McNamara Jr et al, 2012). Cervical vertebrae maturation method was done to assess the skeletal age (Lamparski, 1972). Skeletal age is more accurate than chronological age to evaluate the skeletal maturation of the patients (Baccetti et al, 2005). It was evaluated from the lateral cephalograms hence avoiding the need for additional hand-wrist radiographs. However, it is well-established that intervention with functional appliances at the circumpubertal growth stage yields more skeletal than dental effects, yet, this does not diminish the potential for skeletal growth changes in the postpubertal stage. The amount of skeletal correction in the peak growth study group was $43 \%$ while that of the postpeak group was 25\% (Konik et al, 1997 and Servello et al, 2015).

The SUS 2 is a combination of Herbst and Jasper Jumper which makes it advantageous since it incorporates the advantages of both while avoiding their limitations. It is a telescopic device with a mode of activation different from that of Herbst. It has a telescopic rod which is located inside a guide tube. According to the level of forces needed, the spring inside this tube is activated (Sabbagh, 2006).

A general overview of the effects of correction of Class II in the SUS ${ }^{2}$ study group shows that the 
correction is due to a combination of mild skeletal effects and pronounced dentoalveolar effects. A recent systematic review by McGuinnes, 2016 reported that fixed functional appliances show definite skeletal and dental changes in the short term, even more than removable functional appliances.

Regarding maxillary skeletal changes, a significant sagittal restraint is exerted on the maxilla which results in inhibition of forward maxillary growth or a "headgear effect" with a mean value of $(-0.5 \mathrm{~mm})$ for Pt A to Nasion perp and $\left(-0.89^{\circ}\right)$ for SNA. These results are in accordance with the results of Pancherz, 1982; Valant \& Sinclair, 1989 ; Kucukkeles et al, 2007 and Jones et al, 2008 on fixed functional appliances. Furthermore besides the restraining effect, from a theoretical point of view, a growth modification effect from SUS is validated as it has been reported that it exerts a continuous orthopedic force level upon compression of the spring to $12 \mathrm{~mm}$ which closely matches the previously reported orthopedic force needed for restraint of forward maxillary growth(El-Sheikh et al, 2007). Another possible explanation is the hypothesis of the growth of the skull base which leads to more anterior placement of the Point Nasion while point A remains stationary. This leads to a reduction in angle SNA and confirms the halting action that SUS exerts on forward maxillary growth. Other studies suggested another possible contributing factor to the decrease in SNA angle which is the retroclination of the upper incisors (U1/SN is decreased from $115^{\circ}$ to $105^{\circ}$ ) led to the retraction of point A (Jena et al, 2006) . But the study by Al-Abdwani et al, 2009 had a contradictory theory and stated that changes in incisal inclination due to orthodontic treatment have no clinical relevance to the position of points $\mathrm{A}$ and $\mathrm{B}$.

On the other hand, the results of Weiland and Bantleon, 1995; Weiland et al, 1997; Stucki \& Ingervall, 1998 ; Aras et al, 2011; Oztoprak et al, 2012 and Arora et al, 2018 contradict ours since they reported insignificant effects of fixed functional appliances Forsus and Powerscope on anterior maxillary growth. This could be due to different study designs, growth status methodology and variations in the methods of measurement as well as duration of treatment. Other authors (Heinig \& Goz, 2001 and Jones et al, 2008) also reported no restrictions on forward maxillary growth.

An increase in forward mandibular growth was revealed in the statistically significant difference between $\mathrm{T} 1$ and $\mathrm{T} 2$ regarding angle SNB as well as the linear measurements Pog-NaP and $\mathrm{Co}-\mathrm{Gn}$ perpendicular by the amounts of $1.6 \pm 1.2 \mathrm{~mm}$ and $2.1 \pm 0.9 \mathrm{~mm}$ respectively. This can be explained by the fact that SUS $^{2}$, also known as mandibular propulsor (Hanandeh and El-Bialy, 2010), causes an upward and backward relocation of Articulare during functional treatment (Chen et al, 2002). Condylar growth occurs due to bite jumping and the traction of the muscular and tendinous fibers on the condylar bone surface which stimulates bone remodeling (May et al, 1993; Weiland \& Bantleon, 1995; Weiland et al, 1997 and Stucki \& Ingervall, 1998). However, the extent of horizontal mandibular growth was more significant in their study than ours due to a difference in the mean age of the study groups. In this study the mean age was 2 years older than the mean age in the other studies. According to Pancherz, 1982 the younger the patient, the higher the possibility for enhancement of mandibular growth. In agreement with this study is the research by Arora et al, 2018 who compared between two types of fixed functional appliances in post-pubertal subjects (the Forsus and the Powerscope) and found statistically significant improvements in skeletal mandibular as well as dental measurements with better results rendered by Forsus. The results by Jones et al, 2008 were also in alignment with our study. A skeletal mandibular reaction at such mean age is quite astonishing. Apparently, some mandibular growth potential existed in these cases to explain this reaction. 
Similar results were obtained by similar devices in post-peak pubertal subjects treated by Herbst (Pancherz and Hagg, 1985). Perenetti et al, 2015 reported that fixed functional appliances stimulate mandibular growth but are more effective in prepubertal than post-pubertal subjects.

Contrary to this finding is the study by Kucukkeles et al, 2007 and Jena et al, 2006 who concluded that this increase was not due to actual increase of mandibular body length but was due to a change in the location of the pogonion. Ishaq et al, 2016 in a systematic review based on limited evidence, revealed that fixed functional appliances have little effect on the skeletal mandibular parameters. Servello et al, 2015 in their study of pubertal patients reported that the apical base changes were partly due to the maxillary restraining effects of Forsus and partly because of the increased amount of normal mandibular growth during the peak growth period or CS 3-4. However, our comparisons with control group changes showed that mandibular growth changes were less than those obtained by SUS ${ }^{2}$.

Regarding vertical facial height, mandibular plane angle showed statistically insignificant decrease in SUS $^{2}$ group (P> 0.05). This is in agreement with the results of Pancherz, 1979; Weiland and Bantleon, 1995; Covell et al, 1999 and Nalbantgil et al, 2005 who confirmed that fixed functional appliances do not change the facial height in late adolescent patients. Therefore, they can be used in Class II high angle cases without issues relating to excessive increases in the vertical dimension. Servello et al, 2015 reported a forward or anticlockwise rotation of the mandible and a backward rotation of the occlusal plane which is an effect probably caused by the maxillary restraining effect and the minor intrusion of the upper molars. In Class II elastics group there was a statistically significant increase in mandibular plane angle due to lower molar extrusion. This increased the lower anterior facial height and is therefore contraindicated in high angle cases. No statistically significant changes were observed for the control group.

Part of Class II correction is due to dentoalveolar effects such as retroclination of upper incisors and proclination of the lower incisors. The intrusive and distalizing highpull headgear effect of the appliance acting on the maxillary molar region is transferred to the maxillary incisors through the archwire. This is in agreement with previous studies of Weiland \& Bantleon, 1995 and Weiland et al, 1997. On the mandible it functions by applying an anteriorly and downward directed forces on the mandible with an intrusive and proclining forces on the mandibular incisors. This is due to the mode of attachment being completely on the archwire. These findings are in accordance with those reported in other studies of fixed functional appliances (Heinig \& Goz, 2001; Jones et al, 2008 and Arora et al, 2018). This was not in accordance with the study of Cassidy et al, 2014 who reported more proclination of the lower incisors with the fixed functional appliance compared to Class II elastics. However, their results were not augmented by statistical data. The discrepancy between their results and this study's maybe due to the shorter duration of treatment of SUS ${ }^{2}$ compared to Forsus in their study.

Although the use of intermaxillary elastics for Class II correction is a common method, the major disadvantage is the high need of patient compliance (Aras and Pasaoglu, 2017). Class II elastics correction was due to predominant dentoalveolar effects (Nelson et al, 1999; Combrink et al, 2006; Uzel et al, 2007; Serbesis-Tsarudis and Pancherz, 2008 ) Proclination of lower incisors (Meistrell et al, 1986 and Nelson et al, 1999) and increase in the lower facial height (as much as $5 \mathrm{~mm}$ ) due to molar extrusion are the most noticeable effects. The skeletal effects were negligible and insignificant and comparable to control values due to normal growth. However, in disagreement with the findings of this study, Adams et al, 1972 reported some retraining 
effect of forward maxillary growth exerted by Class II elastics. A viable explanation for the lack of any noticeable skeletal effect for Class II elastics in this study is that the force application may have been affected by patient compliance and thus affects the duration that they are kept worn inside the mouth. It is likely that patients use them only about half of the recommended time. Furthermore Class II elastics exert light forces (73.3grams) (Nelson et al, 1999 and Combrink et al, 2006) which are not enough to induce skeletal changes. The SUS 2 appliance is more rigid than Class II elastics and ensures continuity of the force as it cannot be removed by the patient.

\section{CONCLUSIONS}

This study demonstrates that SUS2 is effective for the treatment of Class II division 1 malocclusion during the young permanent dentition. It has a functional outcome which ceases anterior maxillary growth and induces efficient sagittal mandibular growth in small amounts. These changes may appear small, however, such minor skeletal effects can be associated with a noticeable treatment effect and the success of the treatment outcome.

\section{CLINICAL SIGNIFICANCE}

Measures to reduce lower incisor proclination can be implemented such as lower anterior lingual crown torque or negatively torques lower incisor brackets as well as full ligation of the lower rectangular archwire and reducing the force levels.

Since no significant changes occurred in the vertical facial height measurements, SUS 2 can be used in high angle cases while Class II elastics should not be used due to the increase they induce in the mandibular plane angle.

Even though the skeletal changes induced by SUS2 were mild however they are significant from a clinical perspective as they lead to marked treatment changes and successful treatment outcomes. On the other hand no skeletal changes were induced by Class II elastics but all the effects were dentoalveolar and they exceeded those effected by SUS2. Therefore considering Class II elastics as substitutes for fixed functional appliances for rendering similar effects is not justified.

These amounts are significant compared to Class II elastics which mainly work by inducing more pronounced dentoalveolar changes.

\section{RECOMMENDATIONS}

Long-term follow-up studies are essential to monitor the stability of the skeletal and dental corrections achieved.

\section{REFERENCES}

1. Abd El-Magid M T. Treatment of Developing Class II division 1 Malocclusion with Jasper Jumper. Master thesis, Mansoura University, 1995.

2. Al-Abdwani R1, Moles DR, Noar JH. Change of incisor inclination effects on points A and B. Angle Orthod. 2009 ;79(3):462-7.

3. Adams CP, Meikle MC, Norwick KW, Turpin DL. Dentofacial remodeling produced by intermaxillary forces in Macaca mulatta. Arch Oral Biol 1972; 17(11):1519-35.

4. Aras A, Ada E, Saracoglu H, Gezer N, Aras I. Comparison of treatments with the Forsus fatigue resistant device in relation to skeletal maturity: a cephalometric and magnetic resonance imaging study. Am J Orthod Dentofacial Orthop. 2011;140(5):616-625.

5. Aras I, Pasaoglu A. Class II subdivision treatment with the Forsus Fatigue Resistant Device vs intermaxillary elastics. Angle Orthod. 2017; 87(3):371-376.

6. Arora V, Sharma R, Chowdhary S. Comparative evaluation of treatment effects between two fixed functional appliances for correction of Class II malocclusion:A single-center, randomized controlled trial. Angle Orthod. 2018;88(3):259-266.

7. Baccetti T, Franchi L, McNamara JA Jr. The cervical vertebral maturation method for the assessment of optimal treatment timing in dentofacial orthopedics. Semin Orthod. 2005;11(3):119-129. 
8. Beckwith FR, Ackerman RJ Jr, Cobb CM, Tira DE. An evaluation of factors affecting duration of orthodontic treatment. Am J Orthod Dentofacial Orthop. 1999;115(4):439-447.

9. Bishara SE, Cummins DM, Zaher AR. Treatment and posttreatment changes in patients with Class II, Division 1 malocclusion after ex- traction and nonextraction treatment. Am J Orthod Dentofacial Orthop 1997;111:1827.

10. Buchner HJ. Maintaining mandibular anchorage In Class II, Division 1 treatment. Angle Orthod 1949;19(4):231-49.

11. Cassidy SE, Jackson SR, Turpin DL, Ramsay DS, Spiekerman C, Huang GJ. Classification and treatment of Class II subdivision malocclusions. Am J Orthod Dentofacial Orthop. 2014;145(4):443-451.

12. Chen JY, Will LA, Niederman R. Analysis of efficacy of functional appliances on mandibular growth. Am J Orthod Dentofacial Orthop. 2002; 122(5): 470-6.

13. Combrink FJ, Harris AM, Steyn CL, Hudson AP. Dentoskeletal and soft-tissue changes in growing Class II malocclusion patients during nonextraction orthodontic treatment. SADJ 2006;61(8):344-50.

14. Covell DA Jr, Trammell DW, Boero RP, West R. A cephalometric study of class II Division 1 malocclusion treated with the Jasper Jumper appliance. Angle Orthod. 1999; 69(4): 311-20.

15. Ellen EK, Schneider BJ, Sellke T. A comparative study of anchorage in bioprogressive versus standard edgewise treatment in Class II correction with intermaxillary elastic force. Am J Orthod Dentofa-cial Orthop 1998;114(4):4306.

16. El-Sheikh MM, Godfrey K, Manosudprasit M, Viwattanatipa N. Force-deflection characteristics of the fatigue-resistant device spring: an in vitro study. World J Orthod. 2007;8(1):30-36.

17. Hanandeh BA, El-Bialy AA. Evaluating the effect of Sabbagh Universal Spring during treatment of growing class II malocclusions. Int $\mathrm{J}$ Orthod Milwaukee. 2010;21(4):13-24.

18. Heinig N, Goz G. Clinical application and effects of the Forsus spring. A study of a new Herbst hybrid. J Orofac Orthop. 2001;62(6):436-450.

19. Ishaq RA, AlHammadi MS, Fayed MM, El-Ezz AA, Mostafa Y. Fixed functional appliances with multibracket appliances have no skeletal effect on the mandible: A systematic review and meta-analysis. Am J Orthod Dentofacial Orthop. 2016 ;149(5):612-24.

20. Janson G, Graciano JT, Henriques JF, de Freitas MR, Pinzan A, Pin- zan-Vercelino CR. Occlusal and cephalometric Class II Division 1 malocclusion severity in patients treated with and without extraction of 2 maxillary premolars. Am J Orthod Dentofacial Orthop 2006;129:759-67.

21. Janson G, Sathler R, Fernandes TM, Branco NC, Freitas MR. Correction of Class II malocclusion with Class II elastics: a systematic review. Am J Orthod Dentofacial Orthop. 2013;143(3):383-92.

22. Jena AK, Duggal R, Parkash H. Skeletal and dentoalveolar effects of Twin-block and Bionator appliances in the treatment of Class II malocclusion: a comparative study. Am J Orthod Dentofacial Orthop. 2006;130(5):594-602.

23. Jones G, Buschang PH, Kim KB, Oliver DR. Class II nonextraction patients treated with the Forsus fatigue resistant device versus intermaxillary elastics. Angle Orthod 2008;78(2):332-8

24. Konik M, Pancherz H, Hansen K. The mechanism of Class II correction in late Herbst treatment. Am J Orthod Dentofacial Orthop. 1997;112(1):87-91. .

25. Kucukkeles N, Ilhan I, Orgun IA. Treatment efficiency in skeletal Class II patients treated with the jasper jumper. Angle Orthod. 2007; 77(3): 449-56.

26. Lamparski DG. Skeletal age assessment utilizing cervical vertebrae (dissertation) Master of Science Thesis. 1972 University of Pittsburgh, Pittsburgh.

27. May PW, Chada J, Ledoux WR, Weinberg R, McMinn RW. A cephalometric examination of skeletal and dental changes in Class II, division 1 malocclusion treated with a fixed functional appliance. Certificate Thesis 1993 Louisiana State University School of Dentistry, New Orleans.

28. McGuinness N. Fixed functional appliances show definite skeletal and dental changes in the short term. European Journal of orthodontics. 2016; 38(2):127-128.

29. McNamara JA. Components of class II malocclusion in children 8-10 years of age. Angle Orthod 1981;51(3): 177202 .

30. McNamara JA. A method of cephalometric evaluation. Am J Orthod Dentofacial Orthop. 1984;86(6): 449-469. 
31. McNamara JA Jr, McNamara L, Graber LW. Optimizing orthodontic and dentofacial orthopedic treatment timing. In: Graber LW, Vanarsdall RL, Vig KWL, eds. Orthodontics: Current Principles and Techniques. Philadelphia, Pa: Mosby; 2012:483.

32. Meistrell ME Jr, Cangialosi TJ, Lopez JE, CabralAngeles A. A cephalometric appraisal of nonextraction Begg treatment of Class II malocclusions. Am J Orthod Dentofacial Orthop 1986;90(4): 286-95.

33. Nalbantgil D, Arun T, Sayinsu K, Fulya I. Skeletal, dental and soft-tissue changes induced by the Jasper Jumper appliance in late adolescence. Angle Orthod. 2005; 75(3): 426-36.

34. Nelson B, Hansen K, Hagg U. Overjet reduction and molar correction in fixed appliance treatment of Class II, Division 1, malocclusions: sagittal and vertical components. Am J Orthod Dentofacial Orthop 1999;115(1):13-23.

35. Oztoprak O, Nalbantgil D, Uyanlar A, Arun T. A cephalometric comparative study of class II correction with Sabbagh Universal Spring (SUS2) and Forsus appliances. Eur J Dent. 2012;6(3):302-310.

36. Pancherz H. Treatment of Class II malocclusions by jumping the bite with the Herbst appliance. A cephalometric investigation. Am J orthod 1979;76(4):423-42.

37. Pancherz H. The mechanism of Class II correction in Herbst appliance treatment. A cephalometric investigation. Am J Orthod Dentofacial Orthop. 1982;82(2):104-13.

38. Pancherz H, Hagg U. Dentofacial orthopedics in relation to somatic maturation. An analysis of 70 consecutive cases treated with the Herbst appliance. Am J Orthod Dentofacial Orthop 1985;88(4): 273-287.

39. Perinetti G, Primozic J, Furlani G, Franchi L, Contardo L. Treatment effects of fixed functional appliances alone or in combination with multibracket appliances: a systematic review and meta-analysis. Angle Orthod 2015; 85(3):480-92.

40. Proffit WR. Contemporary Orthodontics. $3^{\text {rd }}$ Edition 1999. C.V. Mosby, 012000. 6.4.2.2.2.

41. Sabbagh A (2006) The Sabbagh Universal Spring. Mosby.

42. Serbesis-Tsarudis C, Pancherz H. "Effective" TMJ and chin position changes in Class II treatment. Angle Orthod 2008;78(5):813-8.

43. Servello DF, Fallis DW, Alvetro L. Analysis of Class II patients, successfully treated with the straight-wire and Forsus appliances, based on cervical vertebral maturation status. Angle Orthod. 2015;85(1):80-86.

44. Skidmore KJ, Brook KJ, Thomson WM, Harding WJ. Factors influencing treatment time in orthodontic patients. Am J Orthod Dentofacial Orthop. 2006;129(2):230-238.

45. Stucki N, Ingervall B. The use of the Jasper Jumper for the correction of Class II malocclusion in the young permanent dentition. Eur J Orthod 1998;20(3):271-81.

46. Uzel A, Uzel I, Toroglu MS. Two different applications of Class II elastics with nonextraction segmental techniques. Angle Orthod 2007;77:694-700.

47. Valant JR, Sinclair PM. Treatment effects of the Herbst appliance. Am J Orthod Dentofacial Orthop. 1989; 95(2): $138-47$.

48. Weiland FJ, Bantleon HP. Treatment of Class II malocclusions with the Jasper Jumper appliance- a preliminary report. Am J Orthod Dentofacial orthop. 1995; 108 (4): $341-50$

49. Weiland FJ, Ingervall B, Bantleon HP, Droschl H. Initial effects of treatment of Class II malocclusion with the Herren activator, activator-headgear combination, and Jasper Jumper. Am J Orthod Dentofac Orthop 1997; 112:19-27. 